\title{
Phase II Study of Irinotecan, 5-Fluorouracil, and Leucovorin in Relapsed or Metastatic Colorectal Cancer as First-line Therapy
}

\author{
Young-Woong Won, M.D., Young-Hyo Lim, M.D., Ho-Yong Park, M.D., Ho-Suk Oh, M.D., Jung-Hye Choi, \\ M.D., Young-Yeul Lee, M.D., In-Soon Kim, M.D., Il-Young Choi, M.D. and Myung-Ju Ahn, M.D \\ Department of Internal Medicine, College of Medicine, Hanyang University, Seoul, Korea
}

Background: The purpose of this study was to assess the efficacy and toxicity of biweekly irinotecan plus 5 -fluorouracil (FU) and leucovorin (LV) in patients with relapsed or metastatic colorectal cancer.

Materials and Methods: Between March 2002 and May 2004, 24 patients with histologically confirmed relapsed or metastatic colorectal cancer were enrolled in this study. One chemotherapy cycle consisted of irinotecan 180 $\mathrm{mg} / \mathrm{m}^{2}$ on days 1 and $15 ; 5-\mathrm{FU} 400 \mathrm{mg} / \mathrm{m}^{2}$ bolus IV with $600 \mathrm{mg} / \mathrm{m}^{2}$ by a 22 hour intravenous infusion on days 1 , 2, 15 and 16 ; and leucovorin $20 \mathrm{mg} / \mathrm{m}^{2}$ on days $1,2,15$ and 16 , every 4 weeks.

Results: The median age of the 24 was 57.5 years (range, $38 \sim 69$ ). Their metastatic sites included: the liver $(62.5 \%)$, lung $(20.8 \%)$, peritone um $(16.7 \%)$, lymph node $(12.5 \%)$, ovary $(8.3 \%)$ and pelvis/vagina $(8.3 \%)$. Twenty. two patients were evaluable for a response. Six and 7 patients achieved partial responses and stable diseases, respectively. The overall response rate was $27.3 \%(95 \%$ Confidence interval; $10.3 \sim 44.5 \%$ ). The median follow-up

\section{INTRODUCTION}

The most widely used agent in the treatment of metastatic colorectal cancer is 5-fluorouracil (5-FU), which was developed more than 40 years ago, and included in most regimens of chemotherapy for colorectal cancer (1). When given alone, as an intravenous bolus, once a week or for five consecutive days every 4-5 weeks, 5-FU produces response rates of between 11 and $17 \%$, with a median survival time of approximately 1 year. Having been the only such drug for a long period of time, much effort has gone into identifying its most efficacious administration. Infusional 5-FU can be considered as the most

Correspondence: Myung-Ju Ahn, Hemato-oncology, Department of Internal Medicine, College of Medicine, Hanyang University, Haengdang-dong, Seongdong-gu, Seoul 133-791, Korea. (Tel) +82-22290-8335, (Fax) +82-2-2298-9183, (E-mail) ahnmj@hanyang. ac.kr Received August 6, 2004, Accepted August 24, 2004 duration for surviving patients was 14.7 months (range, 1 . $7 \sim 26.5$ ). Median overall survival (OS) and 1 -year OS rates were 19 months and $86.3 \%$, respectively. Median response duration and median progression free survival were 7.47 and 5.57 months, respectively. A total of 83 cycles (median 4 cycles) were administered. The main non-hematologic toxicities were nausea/vomiting $(44.5 \%)$ $18.1 \%)$ and diarrhea $(8.4 \%)$. The most common hematologic toxicity was $\mathrm{NCl}$ grade I/II anemia $(31.3 \%)$ and grade I/II neutropenia was $10.8 \%$. There was no life-threatening toxicity.

Conclusion: The results suggested that irinotecan, 5-FU and leucovorin combination chemotherapy in a biweekly schedule is a practical and tolerable treatment option in patients with advanced colorectal cancer. (Cancer Research and Treatment 2004;36:235-239)

Key Words: Irinotecan, 5-Fluorouracil, Leucovorin, Metastatic colorectal cancer

effective and least toxic way to administer 5-FU, which may be combined to achieve the maximum antineoplastic efficacy. A number of biochemical modulators have been used in combination with 5-FU in attempts to improve its efficacy while maintaining acceptable toxicity. The most successful of these to date is folinic acid (FA), with regimens based on 5-FU and FA becoming the standard in the majority of institutions worldwide (2). Although the overall magnitude of increased efficacy may still be considered small, biochemical modulation and infusional 5-FU are increasingly important as combination treatments with other, newer compounds, such as irinotecan and oxaliplatin, especially as the currently available data indicated that not only can the efficacy be improved, but also the toxicity is reduced when these agents are combined with infusional instead of bolus 5-FU (3).

Irinotecan (CPT-11, Campto $\left.{ }^{(}\right)$is a semisynthetic derivative of the plant alkaloid camptothecin, which exerts its cytotoxic activity through the inhibition of the nuclear enzyme topoisomerase I (4). Irinotecan has shown encouraging antitumor activity in a number of solid tumors, including colorectal 
cancer. As a first-line therapy, Rougier et al reported that 48 chemotherapy-naive patients treated with $350 \mathrm{mg} / \mathrm{m}^{2}$ of irinotecan once every 3 weeks achieved an objective response rate of $18.8 \%$ (5). In other single-agent studies, response rates of $23 \sim 32 \%$ were reported $(6 \sim 8)$. Because multidrug therapy with non-cross resistant drugs usually leads to enhanced antitumor activity, compared with single-agent therapy, combining an optimal 5-FU regimen with irinotecan is of strategic priority for the management of patients with advanced colorectal cancer. De Gramont et al reported a simplified bimonthly 5-FU/LV regimen combined with irinotecan (FOLFIRI) as a third-line therapy for patient with advanced colorectal cancer. FOLFIRI consisted of irinotecan $180 \mathrm{mg} / \mathrm{m}^{2}$ as a 90 -min infusion day $1 ; \mathrm{LV} 400 \mathrm{mg} / \mathrm{m}^{2}$ as a 2 -h infusion during irinotecan administration, immediately followed by $5-\mathrm{FU}$ as a $400 \mathrm{mg} / \mathrm{m}^{2}$ bolus and $46-\mathrm{h}$ continuous infusion of $2.4 \sim 3$ $\mathrm{g} / \mathrm{m}^{2}$ every 2 weeks. FOLFIRI generated activity and acceptable toxicity in heavily pretreated patients, with limited diarrhea, mostly asymptomatic neutropenia and manageable nausea and relatively uncommon alopecia (9). Ducreux et al reported a phase I study that combined irinotecan with a bolus of 5-FU, continuous infusion of 5-FU and high dose leucovorin every 2 weeks, in previously treated metastatic colorectal cancer, produced a $22 \%$ response rate, with non-overlapping toxicity (10). This regimen is suitable for studies in chemotherapynaive patients with advanced colorectal cancer.

In the present study, a phase II study was designed to evaluate the efficacy and safety of irinotecan, 5-FU and leucovorin in combination every 2 weeks in relapsed or advanced colorectal cancer.

\section{MATERIALS AND METHODS}

\section{1) Eligibility and patient evaluation}

Histologically or cytologically proven advanced or metastatic colorectal cancer was included. Other eligible criteria included; at least a one- or bidimensional measurable lesion, age $>18$ years, ECOG performance $0^{\sim}$, adequate organ function and a life expectancy of at least 3 months. The patients had to have received no prior chemotherapy for advanced disease. Adjuvant chemotherapy, if administered, should have been completed at least 6 months before enrollment into the trial. Radiotherapy was permitted for palliation, only if the present measurable lesion was not irradiated. The exclusion criteria included; acute serious infection, brain metastasis, abnormal renal or liver function, pregnancy or lactation, serious concomitant illness or medical disease (uncontrolled hypertension, unstable angina, congestive heart failure, acute myocardial infarction within 6 months) and other associated cancer, with the exception of an in situ carcinoma of the cervix and a basal cell carcinoma of skin. This study was approved by the Hanyang University Medical Center Institutional Review Board. All patients gave their written informed consent before enrollment in the study.

\section{2) Treatment schedule}

One chemotherapy cycle consisted of irinotecan $180 \mathrm{mg} / \mathrm{m}^{2}$ on days 1 and $15 ; 5$-FU $400 \mathrm{mg} / \mathrm{m}^{2}$ as an IV bolus, with 600 $\mathrm{mg} / \mathrm{m}^{2}$ as a 22 hour intravenous infusion on days $1,2,15$ and 16 ; and leucovorin $20 \mathrm{mg} / \mathrm{m}^{2}$ on days $1,2,15$ and 16 , every
4 weeks. Treatment was continued until disease progression, unacceptable adverse effects or withdrawal of the patient's consent.

\section{3) Dose modification}

Treatment was delayed after a maximum of 14 days. If there was a second episode of grade 2 or any grade 3 toxicities, the treatment was reduced by $20 \%$. If there was a third occurrence of grade 2, a second episode of grade 3 or any grade 4 toxicities, a $40 \%$ reduction was required. A fourth episode of grade 2, a third episode of grade 3 or a second episode of grade 4 toxicities, despite a dose reduction, the treatment was discontinuation. In the case of myelosuppression, the treatment was postponed or adjusted according to the following instructions; in the case of a $\mathrm{WBC}<4,000 / \mathrm{mm}^{3}$ or platelets $<100,000 / \mathrm{mm}^{3}$ at the start of a cycle, the treatment was postponed for 1 week. If after a maximum delay of 14 days, there was no full recovery, then the following dose reduction should be made.

\section{4) Response criteria and toxicity}

The pretreatment evaluation included a complete medical history and physical examination, complete blood count and chemistry profile measurements, a chest X-ray and a radiological tumor parameter assessment. Patients were assessed for their clinical response after 2 cycles of chemotherapy. The tumor response classification was derived from standard WHO criteria. Toxicities were assessed according to the National Cancer Institute of Canada Clinical Trials Groups' expanded common toxicity grading.

\section{5) Statistical analysis}

The primary end point was the overall objective tumor response rate. The secondary end points were the duration of response, time to disease progression, time to treatment failure and overall survival. The time to disease progression (or progression free survival) was calculated from the date of enrollment to the first observation of a progressive disease or the occurrence of death from any cause. The response duration was calculated from the date of the first documented response to that of progression. This study tested the null hypothesis that 'the true response probability was $\leq 20 \%$ ' against the alternative hypothesis that 'the true probability was $\geq 40 \%$ '. The final decision rule was that at least twelve of the 35 patients needed to experience a response to define the chemotherapy as showing evidence of promising activity. All data were analyzed using SPSS version ${ }^{11.5}$ for windows. A survival curve was obtained using the Kaplan-Meier method.

\section{RESULTS}

\section{1) Patient characteristics and treatment}

Between March 2002 and May 2004, 24 patients were enrolled in this study. Table 1 lists the baseline characteristics for all patients. The median age was $\mathbf{5 7 . 5}$ years, ranging from 38 to 69 years. The primary sites of disease were the colon and rectum in 17 and 7 patients, respectively. The most common site of metastasis was the liver, followed by the lung, peritoneum, lymph node, ovary and pelvis/vagina. Six patients 
Table 1. Baseline characteristics of the study participants $(n=24)$

\begin{tabular}{lrl}
\hline Sex (Male : Female) & $11: 13$ \\
Median age (Range) & 57.5 years $(38 \sim 69$ years) \\
Metastatic site & & \\
Liver & $62.5 \%$ & $(15 / 24)$ \\
Lung & $20.8 \%$ & $(5 / 24)$ \\
Peritoneum & $16.7 \%$ & $(4 / 24)$ \\
Lymph node & $12.5 \%$ & $(3 / 24)$ \\
Ovary & $8.3 \%$ & $(2 / 24)$ \\
Pelivs/Vagina & $8.3 \%$ & $(2 / 24)$ \\
Previous treatment & & \\
Operation & $91.7 \%$ & $(22 / 24)$ \\
Adjuvant chemotherapy & $12.5 \%$ & $(3 / 24)$ \\
Radiation therapy & $8.3 \%$ & $(2 / 24)$ \\
ECOG performance status & & \\
0 & $20.8 \%$ & $(5 / 24)$ \\
1 & $66.7 \%$ & $(16 / 24)$ \\
2 & $12.5 \%$ & $(3 / 24)$ \\
No. of metastatic sites & & \\
1 & $25 \%$ & $(6 / 24)$ \\
2 or more & $75 \%$ & $(18 / 24)$ \\
\hline
\end{tabular}

Table 2. The efficacy of treatment $(n=22)$

\begin{tabular}{lc}
\hline Partial response & $6(27.3 \%)$ \\
Stable disease & $7(31.8 \%)$ \\
Progressive disease & $9(40.9 \%)$ \\
Median response duration & 7.47 months \\
& (range; $1.84 \sim 15.59)$ \\
Median progression & 5.57 months \\
free survival & (range; $1.61 \sim 10.23)$ \\
Median overall survival & 19 months \\
1-year overall survival rate & $86.3 \%$ \\
\hline
\end{tabular}

Table 3. The toxicities of chemotherapy

\begin{tabular}{lcccc}
\hline & \multicolumn{5}{c}{ No. of cycles (n=83) } \\
\cline { 2 - 5 } & Grade 1 & Grade 2 & Grade 3 & Grade 4 \\
\hline Leucopenia & $4(4.8 \%)$ & $5(1.2 \%)$ & $3(3.6 \%)$ & - \\
Neutropenia & $1(1.2 \%)$ & $1(1.2 \%)$ & $4(4.8 \%)$ & $1(1.2 \%)$ \\
Anemia & $13(15.7 \%)$ & $13(15.7 \%)$ & - & - \\
Nausea & $17(20.5 \%)$ & $12(14.5 \%)$ & $8(9.6 \%)$ & - \\
Vomiting & $11(13.3 \%)$ & $1(1.2 \%)$ & $3(3.6 \%)$ & - \\
Diarrhea & $5(6.0 \%)$ & $1(1.2 \%)$ & $1(1.2 \%)$ & - \\
Dermatitis & & & & $1(1.2 \%)$ \\
Stomatitis & & & & $1(1.2 \%)$ \\
\hline
\end{tabular}

(25\%) had one metastatic site, and $18(75 \%)$ had two or more involved organs. All patients had an ECOG performance status between 0 and 2 at the baseline. Most patients (91.7\%) had relapsed colorectal cancer after curative surgery. Three (12.5\%)

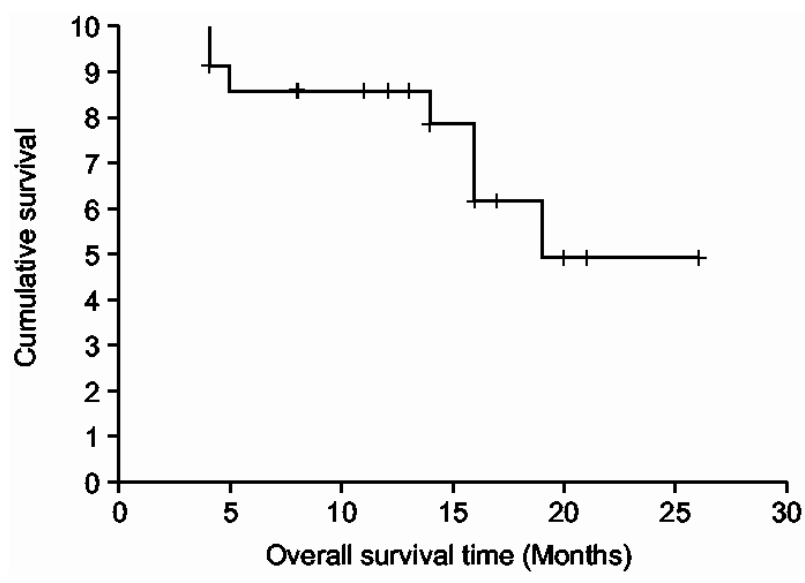

Fig. 1. Kaplan-Meier estimate of overall survival.

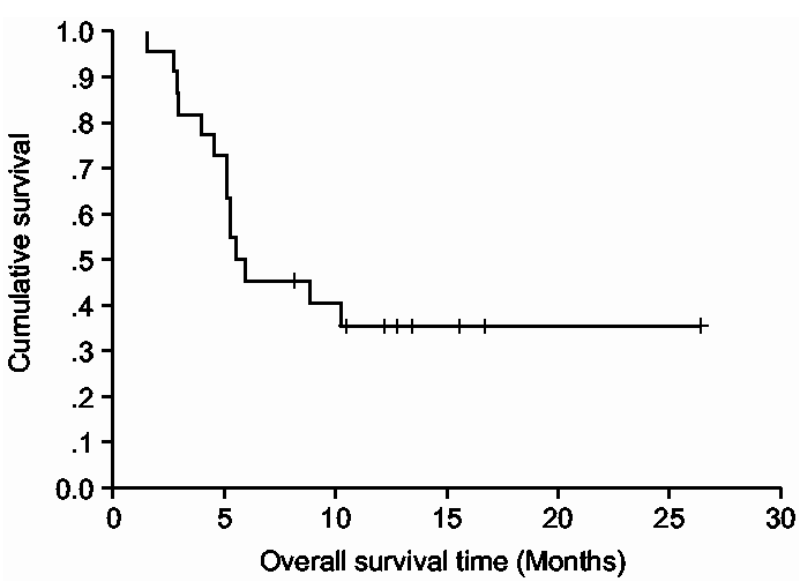

Fig. 2. Kaplan-Meier estimate of time to progression.

and $2(8.3 \%)$ patients had received adjuvant chemotherapy with 5-FU/LV and radiotherapy for palliation, respectively, before enrollment. Two patients were excluded from evaluation; one refused to continue chemotherapy after 4 cycles, and the other could not be evaluated due to having received only 1 cycle of chemotherapy from the time of enrollment. The median relative intensity of the dose of irinotecan and number of treatment cycles administered were $97.9 \%$ and 4 (range, $1 \sim 6$ cycles), respectively. All patients with a progressive disease received further treatment with an oxaliplatin containing regimen.

\section{2) Efficacy}

The results of the response evaluation, assessed by an independent radiological review, are summarized in Table 2. Of the 22 evaluable patients, 6 achieved partial responses and 7 had stable diseases. However, no complete response was observed. The overall response rate was $27.3 \%$ (95\% Confidence interval; $10.3^{\sim}$ 44.5\%). The median follow-up duration of the surviving patients was 14.7 months, ranging from 1.7 to 26.5 months. The median overall survival (OS) and 1-year OS rates were 19 months and $86.3 \%$ (Fig. 1). The median response duration and progression free survival were 7.47 and 
5.57 months, respectively (Fig. 2).

\section{3) Toxicity}

A total of 83 cycles were evaluable for toxicity, and the incidence of toxicity are summarized in Table 3 . The most common hematological toxicities were NCI grade I/II anemia $(31.3 \%)$ and grade I/II neutropenia (10.8\%). Neutropenia reached grade 3 or 4 in 5 cycles (6\%), including 2 patients (2.4\%) who experienced an episode of febrile neutropenia, but did not recur after 5-FU and irinotecan dose reduction in those patients. The main non-hematological toxicities were nausea/ vomiting $(44.5 \% / 18.1 \%)$ and diarrhea $(8.4 \%)$. Grade 3 or 4 nausea and vomiting developed in $8(9.6 \%)$ and 3 cycles (3.6\%), respectively. The other grade 3 or 4 toxicity observed was diarrhea in 1 cycle (1.2\%). There was no life-threatening toxicity.

\section{DISCUSSION}

In previous studies, as a first-line chemotherapy in metastatic colorectal cancer, single-agent irinotecan a produced response rate, median time to progression (TTP) and survival time of $26 \%$ [95\% confidence interval (CI): from $20 \%$ to $32 \%$ ], $8-9$ months and 12 months, respectively $(5,6,8,11)$. Thus, singleagent irinotecan has demonstrated an antitumor efficacy comparable to that of standard FA-modulated 5-FU-based regimens. Furthermore, irinotecan has demonstrated promising antitumor activity in patients with 5-FU-refractory colon cancer, producing response rates in the range of $13 \sim 23 \%$ and median time to progression of $6 \sim 8$ months $(7,12)$.

Based on the promising single-agent activity of irinotecan in the treatment of colorectal cancer, its combination with different 5-FU-based regimens has been investigated. Interesting results, in terms of both efficacy and safety, have been reported for irinotecan in combination with either a standard bolus or an alternating bolus 5-FU/FA schedule, or with a continuous infusional high-dose 5-FU/FA schedule as the first-line therapy $\left(10,13^{\sim} 15\right)$. Two international randomized phase III trials have confirmed the efficacy of irinotecan combined with the most frequently used 5-FU/FA bolus and infusional regimens compared with the corresponding 5-FU/FA alone. The combination arms of both trials demonstrated a significant superiority in terms of efficacy such as, the response rate, median TTP and median survival time, compared with 5-FU/FA alone $(16,17)$. As a result, irinotecan in combination with either a bolus of 5-FU/FA or an infusional 5-FU/FA regimen has been approved as the first-line treatment for patients with advanced or metastatic colorectal cancer, both in the United States and Europe.

In this study, an irinotecan, 5-FU and LV combination chemotherapy regimen for advanced colorectal cancer as the first-line chemotherapy was shown to be effective and safe. The time to progression and median survival time in our study were similar to those of previous studies. However, the overall response rate was somewhat lower than previous studies (16, 17). In another study, of 20 patients evaluable for a response, 8 partial responses were observed, with a response rate of $40 \%$. Six additional patients achieved stable diseases, and six progressed. The median time to progression and median overall survival were 5.0 and 17.3 months, respectively (18). The lower response rate could be explained, in part, by the small number of treatment cycles administered (median, 4 cycles) in this study. Moreover, $31.8 \%$ of stable diseases were observed and these patients were able to be maintained on chemotherapy with this regimen. Therefore, the response rate should be re-evaluated after more chemotherapy cycles in patients with stable diseases.

Nine patients $(40.9 \%)$ showed progression, and since most of these had a good performance status, they were able to receive further treatment with an oxaliplatin containing regimen as a salvage treatment. Three partial responses were observed, with a response rate of $33.3 \%$. Although the response rate was lower than in previous studies, with over $40 \%$ of patients having progression, the overall survival was similar to that of previous results, suggesting that the salvage treatment with oxaliplatin could give rise to an improvement in the overall survival. In recent studies, FA and 5-FU, and irinotecan (FOLFIRI) followed by FA, the 5-FU and oxaliplatin (FOLFOX6) showed a median survival and second progressionfree survival of 21.5 and 14.2 months, respectively (19). In another study, oxaliplatin, 5-FU and FA produced a significantly higher response rate and longer progression-free survival than 5-FU/FA in patients failing irinotecan-based therapies, and as such, was also a useful second-line treatment (20). In an addition study, an irinotecan in combination with 5-FU/LV regimen was safe and effective in oxaliplatin-pretreated advanced colorectal cancer patients. The objective response rate and median progression-free survival and median survival were $20 \%, 24.6$ and 39.6 weeks, respectively (21).

The most frequent adverse events associated with irinotecan include: neutropenia, delayed diarrhea, acute cholinergic syndrome, alopecia, fatigue, and nausea/vomiting (11). In this study, the most common hematological toxicities were anemia and neutropenia. However, neutropenia did not recur after dose reduction. The most common non-hematological toxicities were nausea and vomiting. In a recent study, the most common non-hematological toxicities were nausea (46.8\%) and vomiting $(31.7 \%)$. Diarrhea developed in $18.2 \%$ after each cycle, and the most frequently observed grade $3 \sim 4$ toxicities were neutropenia (18\%) and diarrhea (4.8\%) (18). In addition, another study showed the frequently occurring grade 3 or 4 non-hematological adverse reactions to be nausea/vomiting (10\%), diarrhea (6.7\%) (21). However, only $7.2 \%$ grade 1 or 2 diarrhea was observed in this study. These results suggested that the most common toxicities to be nausea and vomiting. However, other studies have shown diarrhea to be the most common toxicity (16). The overall, toxic effects seen with this irinotecan combination treatment were reversible, non-cumulative and manageable.

\section{CONCLUSIONS}

These results suggested that irinotecan, 5-FU, and LV combination chemotherapy in a biweekly schedule is an effective and tolerable treatment option in the patients with advanced colorectal cancer. 


\section{REFERENCES}

1. Machover D. A comprehensive review of 5-fluorouracil and leucovorin in patients with metastatic colorectal carcinoma. Cancer 1997;80:1179-1187.

2. Advanced Colorectal Cancer Meta-Analysis Project. Modulation of fluorouracil by leucovorin in patients with advanced colorectal cancer: evidence in terms of response rate. J Clin Oncol 1992;10:896-903.

3. Rothenberg ML, Meropol NJ, Poplin EA, Van Cutsem E, Wadler S. Mortality associated with irinotecan plus bolus fluorouracil/leucovorin: summary findings of an independent panel. J Clin Oncol 2001;19:3801-3807.

4. Kunimoto T, Nitta K, Tanaka T, Uehara N, Baba H, Takeuchi M, Yokokura T, Sawada S, Miyasaka T, Mutai M. Antitumor activity of 7-ethyl-10-[4-(1-piperidino)-1-piperidino]carbonyloxy-camptothecin, a novel water-soluble derivative of camptothecin, against murine tumors. Cancer Res 1987;47:5944-5947.

5. Rougier P, Bugat R, Douillard JY, Culine S, Suc E, Brunet P, Becouarn Y, Ychou M, Marty M, Extra JM, Bonneterre J, Adenis A, Seitz JF, Ganem G, Namer M, Conroy T, Negrier S, Merrouche Y, Burki F, Mousseau M, Herait P, Mahjoubi M. Phase II study of irinotecan in the treatment of advanced colorectal cancer in chemotherapy-naive patients and patients pretreated with fluorouracil-based chemotherapy. J Clin Oncol 1997; 15:251-260.

6. Pitot HC, Wender DB, O'Connell MJ, Schroeder G, Goldberg RM, Rubin J, Mailliard JA, Knost JA, Ghosh C, Kirschling RJ, Levitt R, Windschitl HE. Phase II trial of irinotecan in patients with metastatic colorectal carcinoma. J Clin Oncol 1997; 15:2910-2919.

7. Rothenberg ML, Eckardt JR, Kuhn JG, Burris HA 3rd, Nelson J, Hilsenbeck SG, Rodriguez GI, Thurman AM, Smith LS, Eckhardt SG, Weiss GR, Elfring GL, Rinaldi DA, Schaaf LJ, Von Hoff DD. Phase II trial of irinotecan in patients with progressive or rapidly recurrent colorectal cancer. J Clin Oncol 1996;14:1128-1135.

8. Conti JA, Kemeny NE, Saltz LB, Huang Y, Tong WP, Chou TC, Sun M, Pulliam S, Gonzalez C. Irinotecan is an active agent in untreated patients with metastatic colorectal cancer. J Clin Oncol 1996;14:709-715.

9. Andre T, Louvet C, Maindrault-Goebel F, Couteau C, Mabro M, Lotz JP, Gilles-Amar V, Krulik M, Carola E, Izrael V, de Gramont A. CPT-11 (irinotecan) addition to bimonthly, highdose leucovorin and bolus and continuous-infusion 5-fluorouracil (FOLFIRI) for pretreated metastatic colorectal cancer. Eur J Cancer 1999;35:1343-1347.

10. Ducreux M, Ychou M, Seitz JF, Bonnay M, Bexon A, Armand JP, Mahjoubi M, Mery-Mignard D, Rougier P. Irinotecan combined with bolus fluorouracil, continuous infusion fluorouracil, and high-dose leucovorin every two weeks (LV5FU2 regimen): a clinical dose-finding and pharmacokinetic study in patients with pretreated metastatic colorectal cancer. J Clin Oncol 1999;17:2901-2908.

11. Vanhoefer U, Harstrick A, Achterrath W, Cao S, Seeber S, Rustum YM. Irinotecan in the treatment of colorectal cancer: clinical overview. J Clin Oncol 2001;19:1501-1518.
12. Van Cutsem E, Cunningham D, Ten Bokkel Huinink WW, Punt CJ, Alexopoulos CG, Dirix L, Symann M, Blijham GH, Cholet P, Fillet G, Van Groeningen C, Vannetzel JM, Levi F, Panagos G, Unger C, Wils J, Cote C, Blanc C, Herait P, Bleiberg $\mathrm{H}$. Clinical activity and benefit of irinotecan (CPT-11) in patients with colorectal cancer truly resistant to 5-fluorouracil (5-FU). Eur J Cancer 1999;35:54-59.

13. Saltz LB, Kanowitz J, Kemeny NE, Schaaf L, Spriggs D, Staton BA, Berkery R, Steger C, Eng M, Dietz A, Locker P, Kelsen DP. Phase I clinical and pharmacokinetic study of irinotecan, fluorouracil, and leucovorin in patients with advanced solid tumors. J Clin Oncol 1996;14:2959-2967.

14. Rothenberg ML, Cox JV, DeVore RF, Hainsworth JD, Pazdur R, Rivkin SE, Macdonald JS, Geyer CE Jr, Sandbach J, Wolf DL, Mohrland JS, Elfring GL, Miller LL, Von Hoff DD. A multicenter, phase II trial of weekly irinotecan (CPT-11) in patients with previously treated colorectal carcinoma. Cancer 1999;85:786-795.

15. Vanhoefer U, Harstrick A, Kohne CH, Achterrath W, Rustum YM, Seeber S, Wilke H. Phase I study of a weekly schedule of irinotecan, high-dose leucovorin, and infusional fluorouracil as first-line chemotherapy in patients with advanced colorectal cancer. J Clin Oncol 1999;17:907-913.

16. Saltz LB, Cox JV, Blanke C, Rosen LS, Fehrenbacher L, Moore MJ, Maroun JA, Ackland SP, Locker PK, Pirotta N, Elfring GL, Miller LL. Irinotecan plus fluorouracil and leucovorin for metastatic colorectal cancer. N Engl J Med 2000; 343:905-914.

17. Douillard JY, Cunningham D, Roth AD, Navarro M, James RD, Karasek P, Jandik P, Iveson T, Carmichael J, Alakl M, Gruia G, Awad L, Rougier P. Irinotecan combined with fluorouracil compared with fluorouracil alone as first-line treatment for metastatic colorectal cancer: a multicentre randomised trial. Lancet 2000;355:1041-1047.

18. Kim JH, Kim DY, Lee SH, Park SR, Lee SY, Choi IS, Kim TY, Heo DS, Bang YJ, Kim NK. Combination chemotherapy of irinotecan combined with bolus 5-fluorouracil, continuous infusion 5-fluorouracil, and high dose leucovorin every two weeks in recurrent or metastatic colorectal cancer. Korean J Med 2003;64:452-458.

19. Tournigand C, Andre T, Achille E, Lledo G, Flesh M, MeryMignard D, Quinaux E, Couteau C, Buyse M, Ganem G, Landi B, Colin P, Louvet C, de Gramont A. FOLFIRI followed by FOLFOX6 or the reverse sequence in advanced colorectal cancer: a randomized GERCOR study. J Clin Oncol 2004;22:229-237.

20. Rothenberg ML, Oza AM, Bigelow RH, Berlin JD, Marshall JL, Ramanathan RK, Hart LL, Gupta S, Garay CA, Burger BG, Le Bail N, Haller DG. Superiority of oxaliplatin and fluorouracil-leucovorin compared with either therapy alone in patients with progressive colorectal cancer after irinotecan and fluorouracil-leucovorin: interim results of a phase III trial. J Clin Oncol 2003;21:2059-2069.

21. Kwon HC, Kim SH, Kim JS, Kim HJ. Irinotecan combined with bolus fluorouracil, continuous infusion fluorouracil, and low-dose leucovorin every two weeks in patients with oxaliplatin pretreated metastatic colorectal cancer. Cancer Res Treat 2003;35:135-140. 